\title{
LA DESIGUALDAD SALARIAL EN LA INDUSTRIA MANUFACTURERA DE LA FRONTERA DE MÉXICO Y LOS ESTADOS UNIDOS, 1994-2014
}

The wage inequality in the manufacturing industry of the border of Mexico and the United States, 1994-2014 


\section{Resumen}

En este artículo hacemos un análisis comparativo de la desigualdad salarial que existe en la industria manufacturera de los estados que colindan en la zona fronteriza entre México y los Estados Unidos. Para lo cual realizamos un estudio de la desigualdad de los salarios, en los estados del lado mexicano: Baja California, Sonora, Chihuahua, Coahuila, Nuevo león y Tamaulipas; y en los estados del lado estadounidense: California, Arizona, Nuevo México y Texas. Para llevar a cabo este análisis utilizamos la metodología desarrollada por Shorrocks (1982; 2012). De acuerdo con nuestros resultados, encontramos que es sobre todo la desigualdad al interior de los estados la que explica esta reducción de la desigualdad.

Palabras clave: desigualdad salarial, frontera sur de Estados Unidos, frontera norte de México.

\section{Abstract}

In this article we make a comparative analysis of the wage inequality that exists in the manufacturing industry of the neighboring states in the border area between Mexico and the United States. For which we conducted a study of the inequality of wages, in the states of the Mexican side: Baja California, Sonora, Chihuahua, Coahuila, Nuevo Leon and Tamaulipas; and in the US states of: California, Arizona, New Mexico and Texas. To carry out this analysis we use the methodology developed by Shorrocks (1982, 2012). According to our results, we find that, above all inequality within the states is what explains this reduction in inequality.

Keywords: wage inequality, southern border of the United States, northern border of Mexico. 


\section{Introducción}

$\mathrm{L}$ os estados del sur de los Estados Unidos y los estados del norte de México tienen en común el formar parte de la segunda frontera geográfica y económica más extensa del mundo occidental, con la particularidad de que pertenecen a estructuras económicas con niveles de desarrollo diametralmente opuestos. Es importante estudiar, en el contexto geográfico, social y económico de esta zona fronteriza, el comportamiento salarial que determina el nivel de vida que allí prevalece y que contribuye a explicar el comportamiento del mercado laboral de la frontera de México con Estados Unidos.

En este artículo, se hace un análisis comparativo de la desigualdad salarial que existe en la industria manufacturera de los estados que colindan en la zona fronteriza entre México y los Estados Unidos (EE. UU.). Para llevar a cabo este análisis utilizamos la metodología desarrollada por Shorrocks (1982; 2013).

La desigualdad salarial ha sido un tópico ampliamente estudiado en la literatura internacional no sólo por las implicaciones que esta tiene, sino también por la prolongada persistencia del fenómeno a lo largo de las últimas décadas. Y aunque no se haya establecido un consenso sobre la causa principal de esta desigualdad, distintos autores han definido diversos factores que podrían incidir en el aumento de esta, como son: el aumento del trabajo femenino, la apertura comercial, la disminución de la intervención del Estado, el incremento de la oferta laboral (Población Económicamente Activa), la precariedad laboral, el incremento del número de participantes por hogar (esposa e hijos), el cambio tecnológico, factores migratorios, la falta de los sindicatos y la flexibilidad laboral.

La metodología desarrollada por Shorrocks $(1982 ; 2013)$, se basa en la teoría de los juegos cooperativos de Shapley (1953), mediante la cuál se descomponen los índices de Gini de manera exacta, con lo que se evitan los problemas derivados de las mezclas de los ingresos que existen al interior de los subgrupos y que impiden su interpretación correcta. Dicha técnica fue utilizada por Rodríguez, Huesca y Camberos (2011) para analizar la desigualdad salarial entre subgrupos de trabajadores calificados y no calificados y aquellos empleados en áreas tecnológicas y no tecnológicas en México. También, Ochoa, Huesca y Calderón (2015) la utilizaron para estudiar la desigualdad de los salarios entre los trabajadores calificados y no calificados de la frontera norte de México.

La evidencia empírica ha mostrado que, en Estados Unidos, Alemania, y Reino Unido, desde finales de la década de los setentas y principio de los ochenta, se dio una tendencia creciente hacia la desigualdad salarial que se prolongó hasta los primeros años del 2000. En el caso particular de los Estados Unidos, la explican por el aumento de la demanda de trabajo calificado (la elasticidad de sustitución pasó de 1.44 en el año de 1910 a 1.60 para el año 2010) y la reducción de los salarios de los no calificados (Acemoglu y Autor, 2011). Lo anterior coincidió con la revolución tecnológica surgida a partir de la creación, en 1974, del microprocesador. Dicha revolución tuvo como elemento distintivo a la computadora, y en el mercado laboral esta tecnología fue adoptada bajo el esquema de la llamada computarización del lugar de trabajo (Torres y Félix, 2017).

Estos cambios en la estructura salarial se atribuyeron al cambio tecnológico, mismo que provocó una mayor demanda de mano de obra calificada y, por tanto, un incremento en los salarios de este grupo de trabajadores en detrimento del salario de trabajadores no calificados. Estos cambios han provocado 
un aumento en la desigualdad salarial en el citado país que de acuerdo con Calderón, Huesca y Ochoa (2017) el índice de Gini pasó de 0.41 en 2005 a 0.43 en 2012.

Por otro lado, en el caso de México se han observado distintos patrones en el comportamiento de la desigualdad salarial. Previo al año 2000 se mostró un incremento salarial; y posteriormente, desde 2005 hasta 2016, se ha experimentado una reducción de la misma con un índice de Gini que pasó de 0.435 a 0.40. De acuerdo con Huesca y Ochoa (2016) dicha reducción de la desigualdad se debe a una caída en el salario de trabajadores calificados.

Calderón, Huesca y Ochoa (2017) aplicaron la metodología de Shorrocks (1982;2013) para determinar si la Hipótesis de Cambio Tecnológico Sesgado (HCTS) era válida para comprender el comportamiento del mercado laboral de México y realizar un análisis comparativo con el mercado norteamericano. Y se concluyó que la hipótesis antes mencionada tan sólo era válida para la economía estadounidense. Ya que, "esta hipótesis no se verifica en el caso de México, país en vías de desarrollo, pues se advierte que la desigualdad salarial se redujo durante el período de estudio, como resultado de la disminución del salario de los trabajadores más calificados. Entre los factores que explican este fenómeno están el incremento de la oferta de trabajo calificado, la inexistencia de un desarrollo tecnológico endógeno y la precariedad laboral." Calderón, Huesca y Ochoa (2017: 15-16).

Para el análisis de la desigualdad salarial en la industria manufacturera de los estados de la frontera de México y Estados Unidos, este artículo se estructura como sigue: en la primera sección, analizaremos el nivel de vida y desarrollo económico de los estados de la zona fronteriza; en la segunda, la desigualdad económica de la región fronteriza; en la tercera la desigualdad salarial y la población ocupada en México y los EE. UU.; en la cuarta, la desigualdad salarial y la población ocupada en la zona fronteriza de México y EE. UU.; y en la quinta sección, el estudio comparativo de la desigualdad salarial en los estados fronterizos de México y Estados Unidos donde se expone la metodología, los datos utilizados y los resultados.

\section{El Nivel de vida y grado de desarrollo en los estados de la frontera de norte de México y sur de los Estados Unidos.}

En este apartado analizaremos el nivel de vida y el grado de desarrollo de los estados que conforman la frontera México-estadounidense, que como Tamayo y Fernández (1983:11) ya destacaban, pertenecen a dos economías totalmente diferentes, donde el polo dominante se beneficia del polo dominado "En los territorios fronterizos del norte de México y del suroeste de Estados Unidos, particularmente sus localidades fronterizas, se confrontan y articulan consuetudinariamente dos formaciones económico-sociales profundamente diferenciadas. La formación de mayor desarrollo relativo (obvio decirlo, la estadounidense) domina la articulación y se beneficia de la presencia de la otra."

Del lado de México son seis estados los que colindan con los Estados Unidos (Baja California, Sonora, Chihuahua, Coahuila, Nuevo león y Tamaulipas); y del lado de los EE. UU. son cuatro (California, Arizona, Nuevo México y Texas). Comenzaremos por analizar el comportamiento del PIB per cápita y la sigma convergencia entre los estados de esta región fronteriza.

La evolución en el comportamiento del PIB per cápita de los estados del sur de EE. UU. y de los estados del norte de México (cuadro 1), en términos del desarrollo económico, pone en evidencia que el 
nivel de vida de los estados del sur de los EE. UU. está por debajo del promedio nacional de ese país. Mientras que el de los estados del norte de México está por encima del promedio nacional de la república mexicana (gráfica 1).

\section{Gráfica 1. Promedio PIB per cápita México-Estados Unidos 1997-2012}

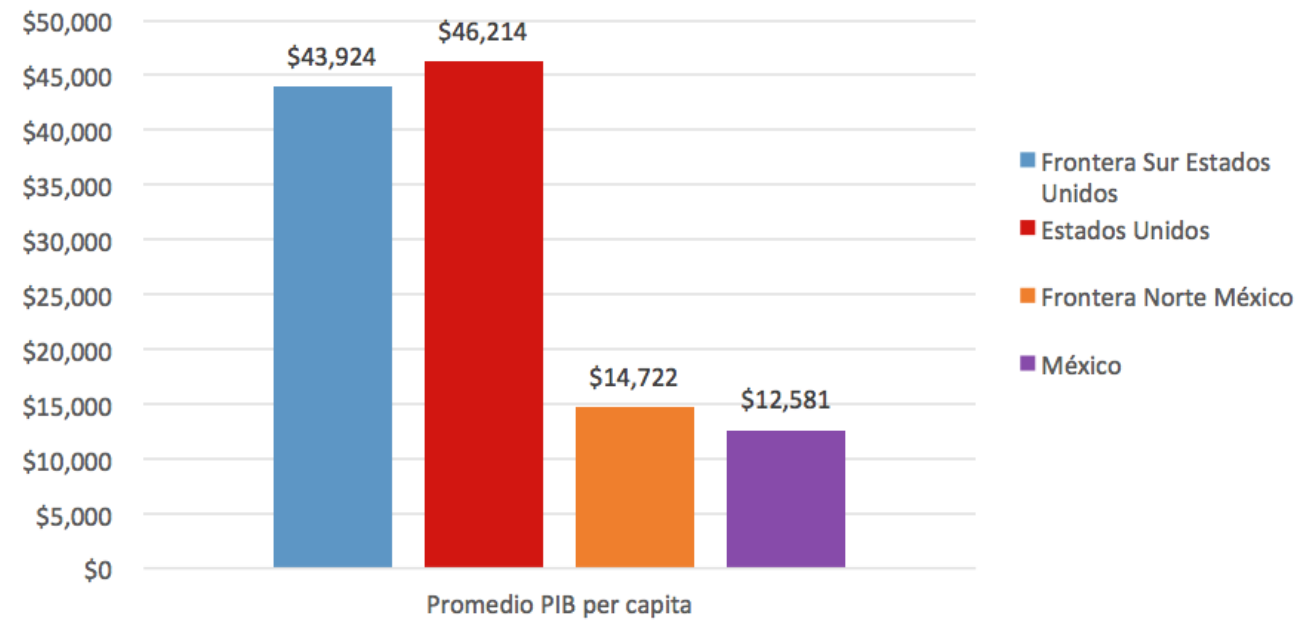

Fuente: Elaboración propia con datos del INEGI, Sistema de Cuentas Nacionales de México, y US Departament of Commerce, Bureau of Economic Analysis

Sin embargo, se observa que, considerados individualmente, los estados de la frontera sur de los EE. UU. más desarrollados son California y Texas, cuyo PIB per cápita se ha mantenido por encima del promedio nacional de ese país; mientras que los estados más subdesarrollados son Arizona y Nuevo México. Asimismo, del lado mexicano, los estados más desarrollados son Nuevo León y Coahuila y los menos desarrollados son Baja California, Sonora, Chihuahua y Tamaulipas. En los estados del sur de los EE.UU., la tasa de variación promedio del PIB per cápita fue mayor en california y Texas, mientras que en México fue mayor en Nuevo León. Por lo que como Anderson y Gerber (2008: 9) señalan

"En los Estados Unidos, la desigualdad del ingreso también es mayor a lo largo de la frontera que a nivel nacional”. Y estas desigualdades también se observan en el caso de la frontera de México.

Pero sin duda como lo señala Anderson y Gerber (2008: 9) "La pobreza es un hecho de la vida en ambos lados de la frontera. Del lado de los EE. UU., la proporción de familias que caen por debajo de la línea oficial de la pobreza es mayor que el promedio nacional" y esto lo corroboramos hasta los años más recientes. 
Cuadro1. PIB per cápita tipo de cambio medido con PPA. México-Estados Unidos y los estados de la frontera. 1997-2012 (dólares)

\begin{tabular}{|c|c|c|c|c|c|c|c|c|c|c|c|c|c|c|}
\hline 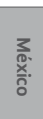 & 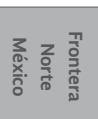 & 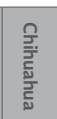 & 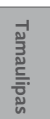 & 高 & 罚 & 送 & 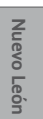 & 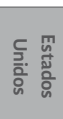 & 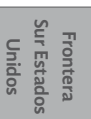 & 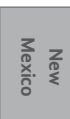 & 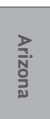 & $\begin{array}{l}\overrightarrow{\vec{D}^{\prime}} \\
\text { 点 }\end{array}$ & 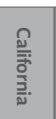 & $\varepsilon$ \\
\hline & & s & 0 & $\infty$ & $v$ & $a$ & u & & & A & $\omega$ & $N$ & $r$ & 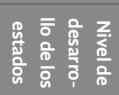 \\
\hline 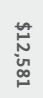 & 莣 & 莣 & $\stackrel{\tilde{E}}{\stackrel{\tilde{\omega}}{\infty}}$ & $\begin{array}{l}\stackrel{\tilde{\omega}}{\omega} \\
\stackrel{\infty}{\infty} \\
\infty\end{array}$ & 莣 & $\begin{array}{l}\tilde{a} \\
\vdots \\
\vdots \\
\vdots\end{array}$ & 芯 & 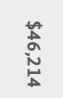 & 莣 & 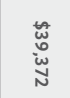 & 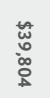 & 蒿 & 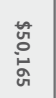 & 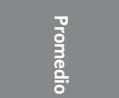 \\
\hline$\stackrel{\sim}{\leftrightarrows}$ & 岕 & 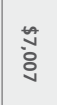 & 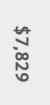 & 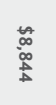 & 蒿 & 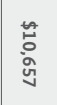 & 莣 & 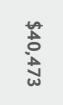 & 芯 & 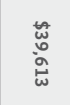 & 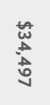 & $\underset{\omega}{\stackrel{\tilde{N}}{\mathbf{W}}}$ & 莣 & $\stackrel{\breve{g}}{\stackrel{0}{0}}$ \\
\hline 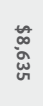 & 莣 & 今̀ & $\begin{array}{l}\stackrel{\leftrightarrow}{\infty} \\
\vdots \\
\vdots\end{array}$ & 芯 & 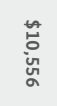 & $\begin{array}{l}\tilde{E} \\
\overrightarrow{5} \\
\text { 去 }\end{array}$ & 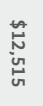 & $\underset{\omega}{\stackrel{\varpi}{*}}$ & 芯 & $\begin{array}{l}\text { 芯 } \\
\text { 品 } \\
\text { ù }\end{array}$ & $\begin{array}{l}\stackrel{\tilde{N}}{\tilde{N}} \\
\stackrel{\tilde{N}}{N}\end{array}$ & 莣 & 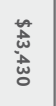 & 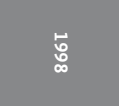 \\
\hline : & 蒿 & 芯 & 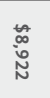 & 莕 & $\tilde{E}$ & 泀 & 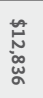 & 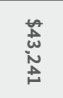 & 芦 & 芯 & 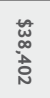 & $\stackrel{\tilde{E}}{\tilde{E}}$ & 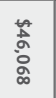 & : \\
\hline $\begin{array}{c}\text { 范 } \\
\text { 心 } \\
\text { v }\end{array}$ & $\stackrel{\tilde{\omega}}{\tilde{\omega}}$ & 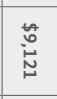 & 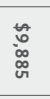 & 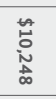 & $\begin{array}{l}\tilde{N} \\
\tilde{E} \\
\tilde{E}\end{array}$ & 芯 & 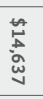 & 蒡 & 芯 & 芯 & 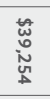 & 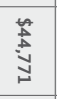 & 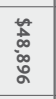 & $\tilde{\Xi}$ \\
\hline . & 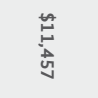 & 荾 & : & 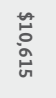 & $\begin{array}{l}\stackrel{*}{\tilde{N}} \\
\vdots \\
0\end{array}$ & 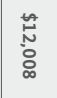 & 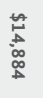 & 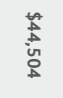 & 芯 & 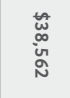 & 芯 & 茅 & 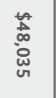 & $\tilde{\text { z }}$ \\
\hline 芦 & 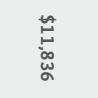 & $\begin{array}{l}\tilde{0} \\
\vdots \\
\infty \\
\infty\end{array}$ & 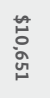 & 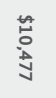 & 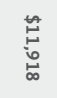 & 芯 & 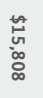 & 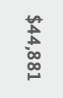 & 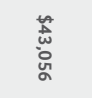 & 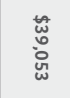 & 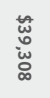 & 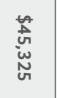 & 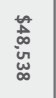 & 芯 \\
\hline 芦 & 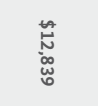 & 芯 & 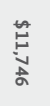 & 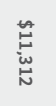 & $\begin{array}{l}\stackrel{n}{N} \\
\text { o. } \\
\text { o. }\end{array}$ & 莣 & 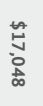 & $\begin{array}{l}\text { 菊 } \\
\text { जै } \\
\text { जै }\end{array}$ & 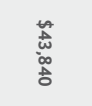 & 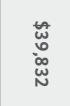 & 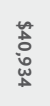 & 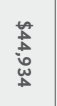 & 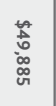 & 莕 \\
\hline 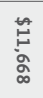 & 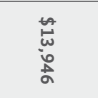 & 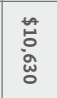 & 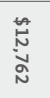 & $\stackrel{\stackrel{\tilde{N}}{\tilde{U}}}{\text { Un }}$ & $\stackrel{\tilde{\tilde{U}}}{\tilde{\sigma}}$ & 芯 & 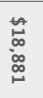 & 苟 & 蓠 & $\begin{array}{l}\text { 芦 } \\
\text { oे }\end{array}$ & 芦 & 荅 & 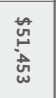 & $\tilde{o}$ \\
\hline 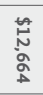 & 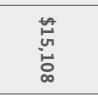 & 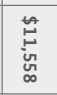 & 芯 & 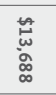 & 苛 & 蒿 & 荅 & 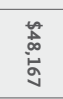 & 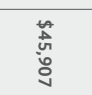 & 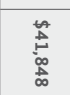 & 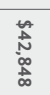 & 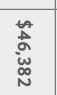 & 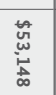 & 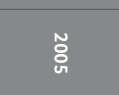 \\
\hline 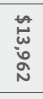 & : & 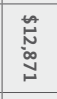 & 菍 & 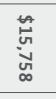 & 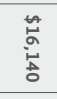 & 芯 & 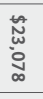 & 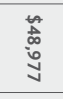 & 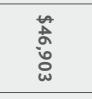 & 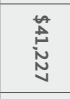 & 菩 & 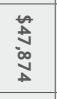 & 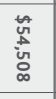 & 听 \\
\hline 莣 & 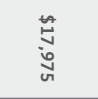 & 芯 & 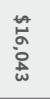 & 蒿 & 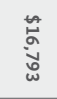 & $\begin{array}{l}\tilde{0} \\
\vdots \\
\vdots \\
\vdots\end{array}$ & 芯 & 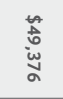 & 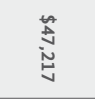 & 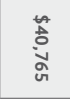 & 莣 & 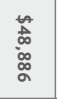 & 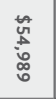 & $\tilde{\sigma}$ \\
\hline 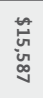 & 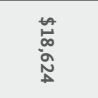 & 莣 & 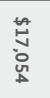 & 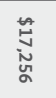 & 莕 & $\begin{array}{l}\tilde{\vdots} \\
\vdots \\
\dot{\sigma}\end{array}$ & 芯 & $\begin{array}{l}\stackrel{+}{\Delta} \\
\stackrel{\Delta}{0}\end{array}$ & 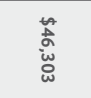 & 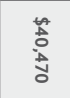 & 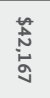 & $\begin{array}{l}\stackrel{+}{\Delta} \\
\stackrel{\tilde{\omega}}{\tilde{\omega}} \\
\end{array}$ & 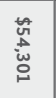 & 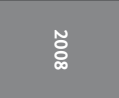 \\
\hline 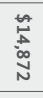 & 芯 & 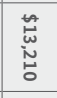 & 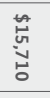 & 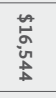 & 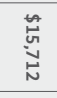 & 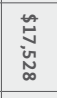 & 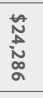 & 苂 & $\underset{\tilde{W}}{t}$ & 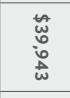 & 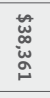 & 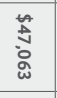 & 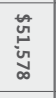 & 行 \\
\hline 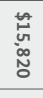 & 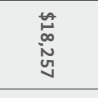 & 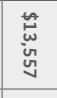 & $\begin{array}{l}\text { 芯 } \\
\text { 岕 } \\
\text { w } \\
\end{array}$ & $\stackrel{\stackrel{\Delta}{N}}{\tilde{N}}$ & 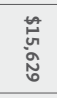 & $\begin{array}{l}\stackrel{\tilde{0}}{0} \\
\dot{0}\end{array}$ & $\begin{array}{l}\text { 芯 } \\
\text { ָू }\end{array}$ & 苛 & 蒡 & $\begin{array}{l}\text { 芯 } \\
\stackrel{\omega}{\omega}\end{array}$ & $\begin{array}{l}\stackrel{\ddot{\omega}}{\tilde{N}} \\
\stackrel{\sim}{N}\end{array}$ & $\begin{array}{l}\tilde{\Delta} \\
\stackrel{\Delta}{v}\end{array}$ & 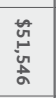 & 蔗 \\
\hline 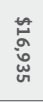 & 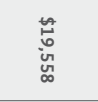 & 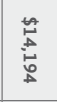 & 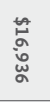 & $\begin{array}{l}\tilde{\tilde{D}} \\
\ddot{\mathbf{E}}\end{array}$ & 芯 & $\begin{array}{l}\stackrel{\tilde{N}}{\tilde{N}} \\
\text { N }\end{array}$ & 芯 & 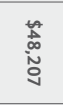 & 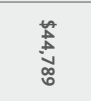 & $\begin{array}{l}\text { 芯 } \\
\text { tै }\end{array}$ & 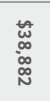 & 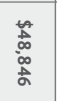 & 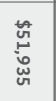 & 䓵 \\
\hline 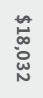 & 莡 & 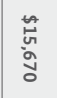 & 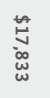 & 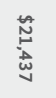 & 芯 & 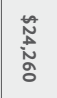 & 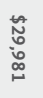 & 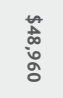 & 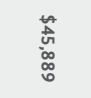 & 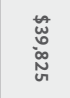 & 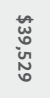 & 芯 &  & T \\
\hline
\end{tabular}

Fuente: Elaboración propia con datos de INEGI Sistema de Cuentas Nacionales de México y US Department of Commerce, Bureau of Economic Analysis. 
Como segundo hecho estilizado, es que, si consideramos únicamente el PIB per cápita, observamos que los estados fronterizos de ambos países tienden a converger. De manera que los niveles de vida, medidos por este indicador, han tendido a converger en el marco del Tratado de Libre Comercio de América del Norte (TLCAN). Lo que, bajo este criterio, significa que los estados del norte de México han tendido a mejorar su nivel de vida, mientras que el nivel de vida de los estados del sur de los EE. UU. ha tendido a disminuir, constatándose que los estados menos beneficiados fueron Nuevo México y Arizona, (véase gráfica 2).

\section{Gráfica 2. Sigma Convergencia en la zona fronteriza entre México y los EE. UU.}

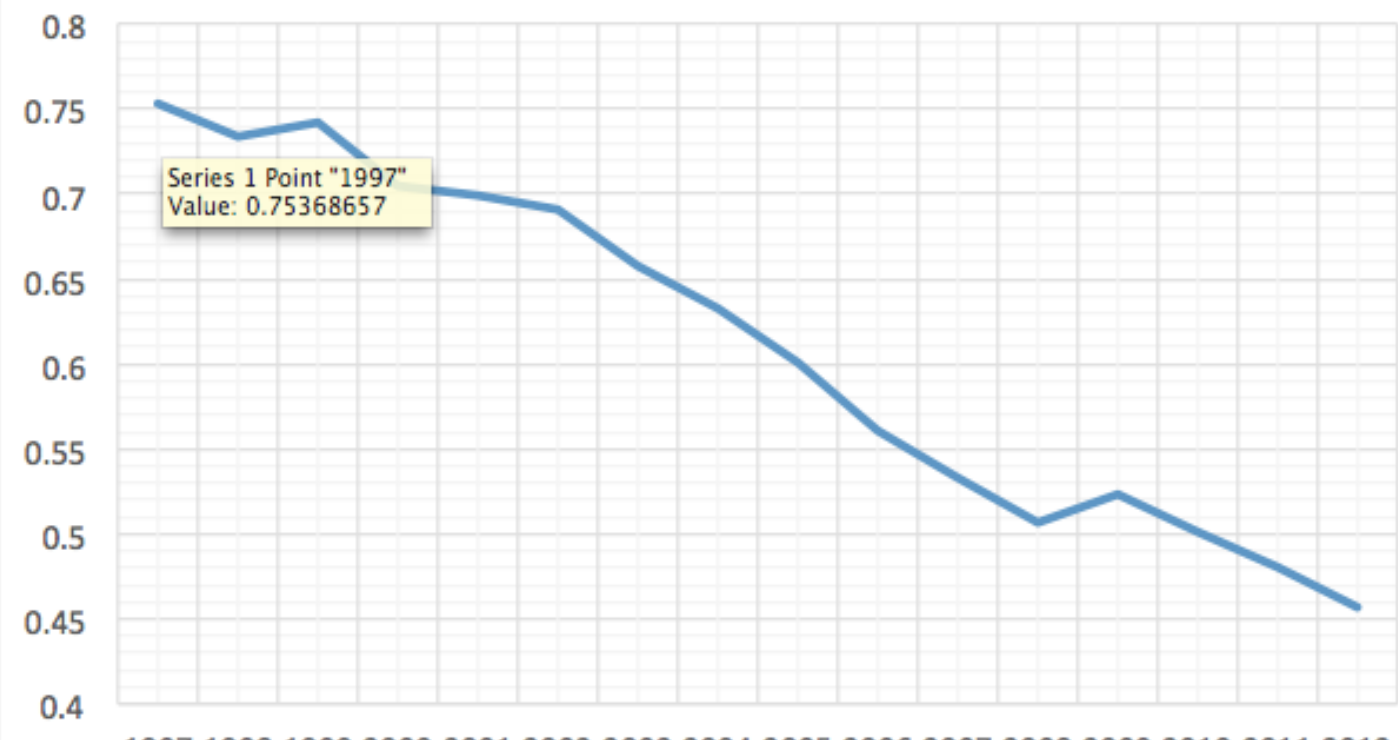

1997199819992000200120022003200420052006200720082009201020112012

Fuente: Elaboración propia con datos de INEGI Sistema de Cuentas Nacionales de México y US Department of Commerce, Bureau of Economic Analysis.

Sin embargo, si sólo tomamos el PIB per cápita como indicador del nivel de vida de la población de la región fronteriza de ambos lados de la frontera, se puede prestar a una serie de confusiones, por lo que es necesario considerar otros indicadores, tales como, el índice de rezago social y el índice de Gini, para poder realizar un análisis más preciso de los niveles de vida de la población y del nivel de desarrollo.

\section{Desigualdad económica en la zona fronteriza.}

A pesar de la tendencia mostrada hacia la convergencia del PIB per cápita en la zona fronteriza, si tomamos en cuenta otros indicadores, existe una brecha muy importante en los niveles de vida de la población de estos estados. En primer término, la convergencia de la región se explica por el hecho que las tasas de crecimiento del PIB per cápita de los cuatro estados de la frontera sur de los Estados Unidos han crecido a tasas inferiores a las de los estados de la frontera norte de México, como se puede observar en los cuadros 2 y 3 . En segundo término, la población de los estados de la frontera sur de EE. UU. es 
- Revista de Ciencias Sociales y Humanidades. ISSN-P: 0188-9834 ISSN-E: 2395-8669.

mayor a la de los estados fronterizos mexicanos. La población de los seis estados de la frontera norte de México, según estimaciones del Consejo Nacional de Población (CONAPO), es de 21,440,654 millones de personas; esto representa el $81 \%$ de la población de Texas, el $56 \%$ de la población de California, y el $29 \%$ de la población total de los cuatro estados fronterizos del sur de los EE. UU. $(73,492,625)$. Cabe añadir que la población de estos cuatro estados fronterizos de EE. UU. representan, entre todos, el 73\% de la población mexicana.

\section{Cuadro 2. Frontera norte de México}

\begin{tabular}{|c|c|c|c|c|c|}
\hline $\begin{array}{l}\text { Entidad Fede- } \\
\text { rativa }\end{array}$ & $\begin{array}{c}\text { Población (Esti- } \\
\text { mación CONAPO } \\
\text { 2014) }\end{array}$ & $\begin{array}{l}\text { Tasa de creci- } \\
\text { miento prome- } \\
\text { dio del PIB per } \\
\text { cápita del período } \\
\text { (1997-2012) }\end{array}$ & $\begin{array}{l}\text { Tasa de creci- } \\
\text { miento promedio } \\
\text { del PIB para el } \\
\text { período (1997- } \\
2012)\end{array}$ & $\begin{array}{l}\text { Esperanza de } \\
\text { vida (2013) }\end{array}$ & $\begin{array}{l}\text { Tasa de escolariza- } \\
\text { ción, promedio de } \\
\text { años de escolaridad } \\
\text { de la población } \\
\text { (2013) }\end{array}$ \\
\hline Baja California & $3,432,944$ & $3.5 \%$ & $5.9 \%$ & \multirow{6}{*}{74.5} & \multirow{6}{*}{8.5} \\
\hline Coahuila & $2,925,594$ & $5.3 \%$ & $6.8 \%$ & & \\
\hline Chihuahua & $3,673,342$ & $5.2 \%$ & $6.8 \%$ & & \\
\hline Nuevo León & $5,013,589$ & $6.2 \%$ & $7.9 \%$ & & \\
\hline Sonora & $2,892,464$ & $5.7 \%$ & $7.4 \%$ & & \\
\hline Tamaulipas & $4,502,721$ & $5.3 \%$ & $7.1 \%$ & & \\
\hline
\end{tabular}

Fuente: Elaboración propia con datos del INEGI, Sistema de

Cuentas Nacionales de México y Proyecciones, CONAPO.

Por otra parte, en términos de la esperanza de vida, en promedio esta es más alta en los estados norteamericanos (78.7 años) que en los estados mexicanos (74.5 años), y el nivel de educación es mayor en los EE. UU. (12.9 años) que en México (8.5 años).

Cuadro 3. Frontera Estados Unidos

\begin{tabular}{|c|c|c|c|c|c|c|}
\hline $\begin{array}{l}\text { Estados } \\
\text { Unidos }\end{array}$ & $\begin{array}{l}\text { Entidad } \\
\text { Federativa }\end{array}$ & $\begin{array}{l}\text { Población } \\
\text { (Estimación } \\
\text { 2013) }\end{array}$ & $\begin{array}{c}\text { Tasa de } \\
\text { crecimiento } \\
\text { promedio del } \\
\text { PIB per cápita } \\
\text { (1997-2012) }\end{array}$ & $\begin{array}{c}\text { Tasa de } \\
\text { crecimiento } \\
\text { promedio del } \\
\text { PIB (1997- } \\
2012\end{array}$ & $\begin{array}{l}\text { Esperanza } \\
\text { de vida } \\
\text { (2012) }\end{array}$ & $\begin{array}{l}\text { Tasa de escolariza- } \\
\text { ción, promedio de } \\
\text { años de escolaridad } \\
\text { de la población } \\
\text { (2013) }\end{array}$ \\
\hline \multirow{4}{*}{$\begin{array}{c}\text { Frontera } \\
\text { Sur }\end{array}$} & Arizona & $6,626,624$ & $0.9 \%$ & $2.9 \%$ & \multirow{4}{*}{78.7} & \multirow{4}{*}{12.9} \\
\hline & California & $38,332,521$ & $1.5 \%$ & $2.5 \%$ & & \\
\hline & $\begin{array}{c}\text { Nuevo Mé- } \\
\text { xico }\end{array}$ & $2,085,287$ & $0.5 \%$ & $1.5 \%$ & & \\
\hline & Texas & $26,448,193$ & $1.4 \%$ & $2.3 \%$ & & \\
\hline
\end{tabular}

Fuente: US Department of Commerce, Bureau of Economic Analysis, U.S. Census Bureau, Population Division, Banco Mundial y UNESCO. 
- Revista de Ciencias Sociales y Humanidades. ISSN-P: 0188-9834 ISSN-E: 2395-8669.

Otro indicador importante de la brecha existente, es el acentuado rezago social de los estados de la frontera norte de México con respecto a los estados del sur de Estados Unidos en materia de servicios públicos de salud y vivienda. En el plano del ingreso, también la desigualdad es mayor en México (cuadro 4).

Cuadro 4. Comparativo de Indicadores de Rezago Social (porcentaje). Frontera México-Estados Unidos 2010

\begin{tabular}{|c|c|c|c|c|c|c|c|}
\hline $\begin{array}{l}\text { Posición } \\
\text { de Reza- } \\
\text { go Social }\end{array}$ & $\begin{array}{c}\text { Entidad Fe- } \\
\text { derativa }\end{array}$ & $\begin{array}{l}\text { Población de } 15 \\
\text { años y más con } \\
\text { educación bási- } \\
\text { ca incompleta } \\
2010 / \text { Sin diplo- } \\
\text { ma de escuela } \\
\text { secundaria } 2010\end{array}$ & $\begin{array}{c}\text { Población } \\
\text { sin derecho } \\
\text { habiencia a } \\
\text { servicios de } \\
\text { salud 2010/ } \\
\text { Sin seguro } \\
\text { de salud } \\
2010\end{array}$ & $\begin{array}{l}\text { Viviendas } \\
\text { que no dis- } \\
\text { ponen de } \\
\text { excusado } \\
\text { o sanitario } \\
2010 / \text { sin } \\
\text { sanitario } \\
2010\end{array}$ & $\begin{array}{l}\text { Viviendas } \\
\text { que no dispo- } \\
\text { nen de dre- } \\
\text { naje 2010/ } \\
\text { instalaciones } \\
\text { incompletas } \\
\text { de drena- } \\
\text { je2010 }\end{array}$ & $\begin{array}{l}\text { Viviendas } \\
\text { que no } \\
\text { disponen } \\
\text { de refri- } \\
\text { gerador } \\
2010 / \text { sin } \\
\text { refrigera- } \\
\text { dor } 2010\end{array}$ & $\begin{array}{l}\text { Coefi- } \\
\text { ciente de } \\
\text { Gini } 2010\end{array}$ \\
\hline 1 & Arizona & 14.6 & 19.1 & 0.1 & 0.2 & 0.3 & 0.455 \\
\hline 2 & $\begin{array}{l}\text { New Mé- } \\
\text { xico }\end{array}$ & 16.5 & 21.4 & 0.1 & 0.1 & 0.1 & 0.464 \\
\hline 3 & California & 17.3 & 19.4 & 0.3 & 0.5 & 1.4 & 0.471 \\
\hline 4 & Texas & 18.9 & 24.6 & 0.5 & 0.9 & 1.1 & 0.469 \\
\hline 5 & $\begin{array}{l}\text { Nuevo } \\
\text { León }\end{array}$ & 28.3 & 20.3 & 2.3 & 2.6 & 5.6 & 0.498 \\
\hline 6 & Coahuila & 32.5 & 21.9 & 2.1 & 4.3 & 5.7 & 0.477 \\
\hline 7 & Sonora & 32.7 & 25.0 & 2.9 & 9.7 & 8.0 & 0.479 \\
\hline 8 & $\begin{array}{l}\text { Baja Cali- } \\
\text { fornia }\end{array}$ & 35.0 & 28.8 & 2.1 & 5.8 & 8.0 & 0.506 \\
\hline 9 & Chihuahua & 40.2 & 23.5 & 3.5 & 6.5 & 8.7 & 0.473 \\
\hline 10 & $\begin{array}{l}\text { Tamauli- } \\
\text { pas }\end{array}$ & 35.9 & 22.5 & 2.6 & 11.5 & 10.4 & 0.450 \\
\hline
\end{tabular}

Fuente: Elaboración propia. Datos para México estimaciones CONEVAL. Datos para Estados Unidos U.S. Census Bureau, Current Population Survey, Annual Social and Economic Supplement.

La población en situación de pobreza es más alta en los cuatro estados de la frontera sur de los EE. UU., es decir, está por encima del promedio nacional de los EE. UU. (15\%), pero, está muy por debajo de la población en condición de pobreza de los estados fronterizos mexicanos (cuadro 5). Por lo que a pesar de la relativa bonanza en términos del ingreso de los estados del norte de México con respecto al resto del país, comparado con los estados más pobres de la frontera de EE.U. los del lado mexicano son aún más pobres "las tasas de pobreza en el lado mexicano son más bajas que el promedio nacional pero más altas que las del lado de los EE. UU., Y siguen siendo un gran problema” Anderson y Gerber (2008: 9)

A pesar de la convergencia observada entre los estados de la zona fronteriza, en términos del comportamiento del PIB per cápita, se constata que existe una divergencia creciente en los niveles de desarrollo entre los estados del norte de México y los estados del sur de EE.UU. 
Cuadro 5. Estados Unidos, 2010 y 2012 Personas en Situación de Pobreza (todos excepto personas menores de 15)

\begin{tabular}{|c|c|c|c|c|}
\hline \multirow{2}{*}{ Situación de Pobreza en 2010 y 2012} & \multicolumn{2}{|c|}{2010} & 2012 \\
\cline { 2 - 5 } & En Pobreza & No en Pobreza & En Pobreza & No en Pobreza \\
\hline Arizona & $18.80 \%$ & $81.20 \%$ & $19.00 \%$ & $81.00 \%$ \\
\hline California & $16.30 \%$ & $83.70 \%$ & $15.90 \%$ & $84.10 \%$ \\
\hline New Mexico & $18.30 \%$ & $81.70 \%$ & $20.40 \%$ & $79.60 \%$ \\
\hline Texas & $18.40 \%$ & $81.60 \%$ & $17.00 \%$ & $83.00 \%$ \\
\hline Nacional & $15.10 \%$ & $84.90 \%$ & $15.00 \%$ & $85.00 \%$ \\
\hline
\end{tabular}

Fuente: Elaboración propia con datos del U.S. Census Bureau, Current Population Survey,

Annual Social and Economic Supplement.

"En México, el desarrollo se ha visto obstaculizado desde hace mucho tiempo por una distribución del ingreso muy desigual, (...) (y esto a pesar de que) la distribución del ingreso en los estados de la frontera mexicana es más equitativa y el ingreso familiar medio es más alto que en todo México. Anderson y Gerber (2008: 9)

"En los Estados Unidos, la desigualdad del ingreso también es mayor a lo largo de la frontera que a nivel nacional". Anderson y Gerber (2008: 9)

\section{Desigualdad salarial y población ocupada en México y Estados Unidos}

Si analizamos la estructura ocupacional de ambas economías durante el período del TLCAN, cuadro 6 y gráfica 3, se observa que los EE. UU. es el país donde el sector manufacturero absorbió a un número creciente de trabajadores. Es decir, el TLCAN benefició al empleo en este sector estratégico; el cuál a su vez, impulsó el crecimiento de la economía de los EE. UU. desde la década de los noventas hasta el 2007. Cabe destacar que este crecimiento en los EE. UU. está estrechamente relacionado con el crecimiento del sector manufacturero en México, que es complementario al estadounidense. Es decir, por efecto del TLCAN, en la medida que aumenta la producción del sector manufacturero en los EE. UU. aumentan las armadoras e industrias maquiladoras en México, con la consecuente ocupación de mano de obra barata en el sector mexicano. 
Gráfica 3. Personal ocupado en la industria manufacturera en México y los EE. UU.

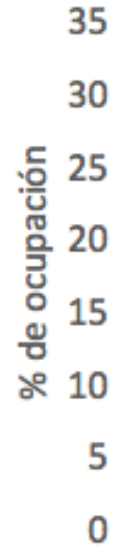

México

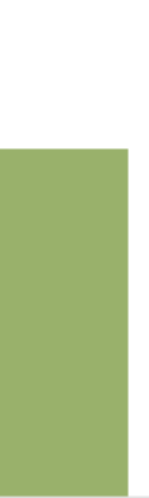

Estados unidos

Industria manufacturera

= $1994=2014$

Fuente: Elaboración propia con datos de ENOE y del U.S. Census Bureau, Current Population Survey

Otro rasgo importante es que los EE. UU. se industrializaron durante este período, ya que la población ocupada en el sector pasó de $24 \%$ a $33 \%$, el comercio se redujo del 23\% al 7.6\%, y los servicios apenas crecieron. En contraste, en México el sector comercio y servicios tuvo una hipertrofia, ya que concentró al $61 \%$ de la población ocupada, con lo cual se observa una absorción espuria ${ }^{4}$ de la fuerza laboral por este sector (cuadro 6).

Cuadro 6. Población ocupada por sector de actividad en México y Estados Unidos

\begin{tabular}{|c|c|c|c|c|c|c|c|c|}
\hline \multirow{2}{*}{$\begin{array}{l}\text { Sector de } \\
\text { actividad }\end{array}$} & \multicolumn{4}{|c|}{1994} & \multicolumn{4}{|c|}{2014} \\
\hline & Estados Unidos & $\%$ & México & $\%$ & Estados Unidos & $\%$ & México & $\%$ \\
\hline Construcción & $60,740,301$ & 0.6 & 660,817 & 4.56 & 42900000 & 0.33 & 6671669 & 14.21 \\
\hline Manufactura & 2428529014 & 24.14 & 673,108 & 4.65 & 4350000000 & 33.18 & 11400000 & 24.29 \\
\hline Comercio & 2374639066 & 23.6 & 4919901 & 33.96 & 978000000 & 7.46 & 28600000 & 60.93 \\
\hline Servicios & 5197255838 & 51.66 & 8234488 & 56.84 & 7740000000 & 59.03 & 263963 & 0.56 \\
\hline Total & $10,061,164,219$ & 100 & $14,488,314$ & 100 & 13110900000 & 100 & 46935632 & 100 \\
\hline
\end{tabular}

Fuente: Elaboración propia con datos de ENOE y del U.S. Census Bureau, Current Population Survey

4- De acuerdo a Prebisch (1971) es la población que expulsa el campo y que, por la insuficiencia de la industria, esta no la emplea y termina absorbiéndola el sector servicios. 
En términos de los salarios promedio se observa una tendencia opuesta entre ambos países, mientras que los salarios de los trabajadores mexicanos se redujeron de manera sensible, el de los trabajadores norteamericanos aumentó sustancialmente en todos los sectores. Durante la vigencia del TLCAN los trabajadores mexicanos perdieron y los americanos se beneficiaron en este rubro (cuadro 7).

\section{Cuadro 7. Salario por hora por sector de actividad}

\begin{tabular}{|c|c|c|c|c|}
\hline \multirow{2}{*}{ Sector de actividad } & \multicolumn{2}{|c|}{1994} & \multicolumn{2}{c|}{2014} \\
\cline { 2 - 5 } & Estados Unidos & México & Estados Unidos & \multirow{2}{*}{ México } \\
\hline Construcción & 9.83 & 4.28 & 21.26 & 1.29 \\
\hline Manufactura & 13.62 & 3.94 & 25.99 & 2.82 \\
\hline Comercio & 9.11 & 3.73 & 28.73 & 2.82 \\
\hline Servicios & 12.14 & 3.96 & 23.02 & 0.94 \\
\hline
\end{tabular}

Fuente: Elaboración propia con datos de ENOE y del U.S. Census Bureau, Current Population Survey

\section{Desigualdad salarial y población ocupada en la zona fron- teriza de México y Estados Unidos.}

En este apartado analizaremos la desigualdad salarial y la estructura ocupacional que prevalece en la zona fronteriza México-EE. UU. En primer término, haremos un análisis comparativo de las estructuras ocupacionales. Y, en segundo término, haremos una comparación de las tendencias salariales observadas en los estados de la frontera norte de México y sur de los EE. UU.

En términos de género, entre 1994 y el 2014, en la frontera norte de México (FNM) la ocupación cambió de manera sustantiva. Ya que tendió a incrementarse la ocupación de los trabajadores hombres y a disminuir el trabajo femenino. El grado de calificación de toda la mano de obra tendió a mejorar, sobre sobre todo en el nivel de secundaria completa y preparatoria (cuadro 8).

Cuadro 8. Composición del empleo en la frontera norte de México

\begin{tabular}{|c|c|c|}
\hline \multicolumn{3}{|c|}{ Frontera Norte de México } \\
\hline Variables & 1994 & 2014 \\
\hline & $\%$ & $\%$ \\
\hline Hombre & Género & \\
\hline Mujer & 48.83 & 62.38 \\
\hline Total & 51.17 & 37.62 \\
\hline
\end{tabular}




\begin{tabular}{|c|c|c|}
\hline \multicolumn{3}{|c|}{ Nivel de instrucción } \\
\hline Primaria completa & 37.13 & 20.5 \\
\hline Secundaria completa & 30.14 & 32.1 \\
\hline Preparatoria & 18.2 & 24.36 \\
\hline Universidad & 6.2 & 12.09 \\
\hline Posgrado & 8.34 & 10.95 \\
\hline Total & 100 & 100 \\
\hline
\end{tabular}

Fuente/ Elaboración propia con datos de la Encuesta Nacional de Empleo Urbano y Encuesta Nacional de Ocupación y Empleo, INEGI,

En el mismo período, en los estados de la frontera sur de los EE. UU., se observaron tendencias similares: incremento del trabajado masculino y reducción del femenino. Y los niveles de calificación del trabajo aumentaron en los niveles universitario y de posgrado (cuadro 9).

Cuadro 9. Composición del empleo en la frontera sur de EE.UU.

\begin{tabular}{|c|c|c|}
\hline \multicolumn{3}{|c|}{ Frontera Sur de Estados Unidos } \\
\hline \multirow{2}{*}{ Variables } & 1994 & 2014 \\
\hline & $\%$ & $\%$ \\
\hline \multicolumn{3}{|c|}{ Género } \\
\hline Hombre & 54.708 & 55.68 \\
\hline Mujer & 45.292 & 44.32 \\
\hline Total & 100 & 100 \\
\hline \multicolumn{3}{|c|}{ Nivel de instrucción } \\
\hline Primaria completa & 15.036 & 11.89 \\
\hline Secundaria completa & 28.077 & 24.89 \\
\hline Preparatoria & 31.262 & 29.15 \\
\hline Universidad & 17.372 & 22.57 \\
\hline Posgrado & 8.253 & 11.51 \\
\hline Total & 100 & 100 \\
\hline
\end{tabular}

Fuente: Elaboración propia con datos del U.S. Census Bureau, Current Population Survey

En términos sectoriales, en los estados de la frontera norte de México, el sector manufacturero (la industria maquiladora de exportación) en el 2014 incrementó su participación en la generación de empleo, mientras que el comercio y los servicios disminuyeron, (cuadro 10). 
Cuadro 10. Porcentaje de ocupación por sector de actividad en los estados de la Frontera Norte de México

\begin{tabular}{|c|c|c|c|c|}
\hline 1994 & Construcción & Manufactura & Comercio & Servicios \\
\hline Baja California & 4.33 & 6.56 & 36.99 & 52.13 \\
\hline Coahuila & 3.03 & 9.41 & 33.63 & 53.93 \\
\hline Chihuahua & 2.93 & 2.91 & 43.88 & 50.29 \\
\hline Nuevo León & 3.4 & 6.68 & 32.64 & 57.29 \\
\hline Sonora & 2.69 & 3.51 & 35.98 & 57.81 \\
\hline Tamaulipas & 4.65 & 2.22 & 39.7 & 53.43 \\
\hline Baja California & & & & 42.18 \\
\hline Coahuila & 6.6 & 28.54 & 22.69 & 43.05 \\
\hline Chihuahua & 8.68 & 29.62 & 18.65 & 43 \\
\hline Nuevo León & 8.71 & 31.74 & 20.06 & 39.48 \\
\hline Sonora & 9.01 & 24.64 & 19.15 & 47.2 \\
\hline Tamaulipas & 8.61 & 19.31 & 23.5 & 48.58 \\
\hline
\end{tabular}

Fuente: elaboración propia con datos de la Encuesta Nacional de Empleo Urbano y Encuesta Nacional de Ocupación y Empleo, INEGI.

En los estados de la Frontera Sur de los EE. UU. los sectores de la manufactura y servicios ocuparon a más trabajadores, y la ocupación en el sector comercio tendió a disminuir, (cuadro 11).

Cuadro 11. Porcentaje de ocupación por sector de actividad en los estados de la frontera sur de EE. UU.

\begin{tabular}{|c|c|c|c|c|}
\hline 1994 & Construcción & Manufactura & Comercio & Servicios \\
\hline Arizona & 0.58 & 18.35 & 25.39 & 55.68 \\
\hline California & 0.51 & 22.42 & 24.09 & 52.97 \\
\hline $\begin{array}{c}\text { Nuevo Mé- } \\
\text { xico }\end{array}$ & 0.24 & 11.62 & 28.02 & 60.12 \\
\hline Texas & 0.53 & 22.92 & 24.12 & 52.43 \\
\hline Arizona & 0 & & & \\
\hline California & 0.22 & 31.54 & 9.34 & 59.12 \\
\hline $\begin{array}{c}\text { Nuevo Mé- } \\
\text { xico }\end{array}$ & 0 & 32.62 & 7.06 & 60.1 \\
\hline Texas & 0.37 & 21.12 & 5.09 & 73.79 \\
\hline
\end{tabular}

Fuente: Elaboración propia con datos del U.S. Census Bureau, Current Population Survey 
Por otra parte, si realizamos el análisis comparativo en términos del salario promedio, se observa un incremento sustancial de la brecha salarial entre 1994 y el 2014. Por lo que los diferenciales salariales entre los estados fronterizos de ambos países crecieron y no disminuyeron como lo pronosticaron los artífices del TLCAN. Este diferencial salarial es un factor que favoreció el flujo migratorio de México hacia los EE. UU.

\section{Cuadro 12. Salario promedio por hora en la FNM}

\begin{tabular}{|c|c|c|}
\hline \multirow{2}{*}{ Frontera Norte de México } & \multicolumn{2}{|c|}{} \\
& \multicolumn{2}{|c|}{ Salario promedio por hora ajustado por PPA } \\
\hline Baja California & 1994 & 2014 \\
\hline Coahuila & 3.65 & 1.29 \\
\hline Chihuahua & 3.599 & 1.56 \\
\hline Nuevo León & 1.28 & 1.28 \\
\hline Sonora & 4.958 & 1.29 \\
\hline Tamaulipas & 5.671 & 1.66 \\
\hline & 3.371 & 1.23 \\
\hline
\end{tabular}

Fuente: Elaboración propia con datos de la Encuesta Nacional de Empleo Urbano y Encuesta Nacional de Ocupación y Empleo, INEGI,

En los estados de la frontera norte de México se observa una fuerte tendencia hacia la reducción de los salarios promedio entre 1994 y el 2014, y una caída en promedio del 100\% del poder de compra de los asalariados de estos estados (cuadro 12). Mientras que los salarios de los estados del sur de los EE. UU. se incrementaron de manera sustancial, sobre todo en Arizona y en Nuevo México. Por lo que entre los estados de México y los EE.UU. se observan tendencias opuestas en términos salariales, dado que tendieron a aumentar en los estados de la frontera sur de los EE. UU. y a reducirse de manera dramática en los estados de la frontera norte de México (cuadro 14).

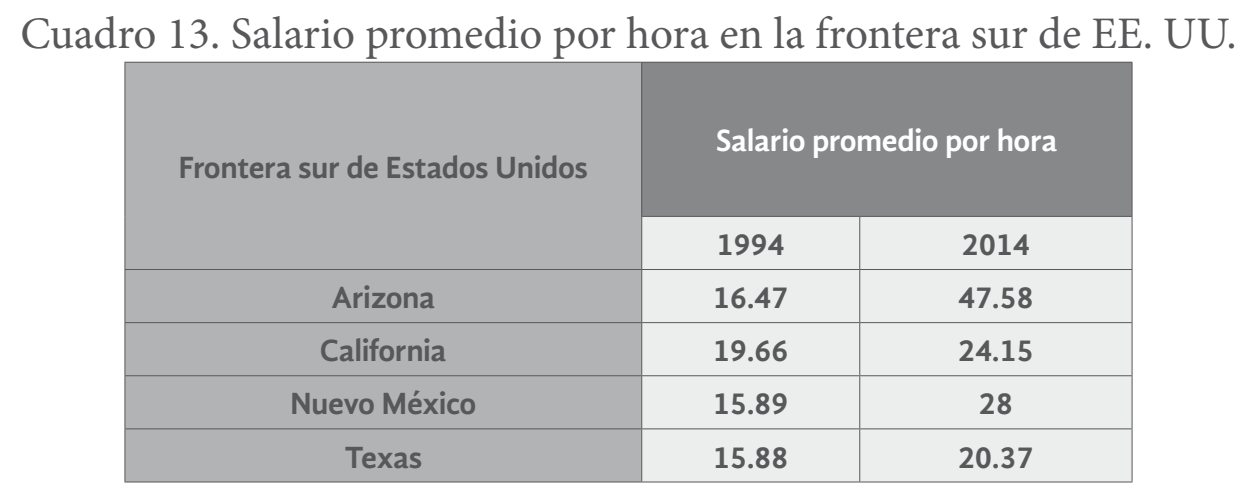

Fuente: Elaboración propia con datos del U.S. Census Bureau, Current Population Survey 


\section{Estudio comparativo de la desigualdad salarial en los estados fronterizos de México y Estados Unidos.}

Procederemos ahora, mediante la metodología de Shorrocks (1982; 2013), a hacer el estudio comparativo de la desigualdad de los salarios en la industria manufacturera de los estados fronterizos de México y los Estados Unidos (EE. UU.),

\subsection{Datos utilizados.}

En el presente documento se utilizaron las bases de datos de la Encuesta Nacional de Empleo Urbano (ENEU) para el año 1994, y para el 2014 se utilizó la Encuesta Nacional de Ocupación y Empleo $(\mathrm{ENOE})^{5}$, los datos de Estados Unidos se obtienen de la Current Population Survey (CPS) para cada uno de los años analizados. La unidad de análisis son los seis estados de la frontera norte de México (Baja California, Sonora, Chihuahua, Coahuila, Nuevo León y Tamaulipas) y los cuatro estados de la frontera sur de Estados Unidos (Arizona, California, Nuevo México y Texas). Asimismo, utilizamos la información correspondiente a la Población Económicamente Activa (PEA) ocupada en México y Estados Unidos y que trabaja en la industria manufacturera en la frontera entre ambos países.

\subsection{Metodología.}

El coeficiente de Gini es uno de los más utilizados para el análisis de desigualdad, este coeficiente acota intervalos de valores entre cero y uno, donde el cero es el valor más cercano a la igualdad y el uno a la más desigual. Sin embargo, de acuerdo con Shorrocks (1982), una de las propiedades de un buen índice de desigualdad es que, se pueda descomponer y sea aditivo. Y precisamente, la deficiencia que presenta el índice de Gini al ser descompuesto, es que mantiene un residuo que no se puede interpretar, el cuál proviene del traslape de los ingresos entre los subgrupos de la distribución. Y que, además, produce una descomposición no exacta entre los componentes "intra" y "entre" del Índice (Bhattacharya y Mahalanobis, 1967).

Se retoma la metodología utilizada por Calderón, Huesca y Ochoa (2017), quienes utilizan el método planteado por Shorrocks (2013), quien realiza una descomposición exacta del índice de Gini mediante la aplicación de Shapley (1953).

Bajo dicha metodología existen aplicaciones a nivel internacional para estudiar la desigualdad y la pobreza (Kolenikov y Shorrocks, 2005; Morduch y Sicular, 2002). Para el caso de México estas aplicaciones se utilizan para analizar el comportamiento de los grupos de trabajadores calificados y no calificados, grupos que realizan tareas en el sector formal e informal, y para los trabajadores de estados de la frontera

norte de México (Rodríguez, Huesca y Camberos, 2011); (Ochoa, Huesca y Calderón 2015); (Huesca y Ochoa, 2016) y (Calderón, Huesca y Ochoa 2017).

5- INEGI emitió la ENEU de 1987 a 2004, posteriormente realiza modificaciones y emite la nueva encuesta ENOE del año 2005 hasta el más reciente segundo trimestre de 2017. 
La descomposición utilizada se basa en la teoría de juegos cooperativos, en la que se obtiene una participación exacta de cada individuo. Mediante la solución de Shapley se asigna a cada trabajador su contribución marginal promedio sobre las combinaciones entre los grupos participantes. En el presente artículo la descomposición se realiza para los seis estados de la frontera norte de México y para los cuatro estados de la frontera sur de los Estados Unidos.

Para el caso de los seis estados de la frontera norte de México, se descompone la desigualdad de la siguiente manera:

$$
\begin{aligned}
& G_{1}^{S}=0.166\left[\mu-\mu_{6}-\mu_{5}-\mu_{4}-\mu_{3}-\mu_{2}-\mu_{1}\right] \\
& G_{2}^{S}=0.166\left[\mu-\mu_{6}-\mu_{5}-\mu_{4}-\mu_{3}-\mu_{1}-\mu_{2}\right] \\
& G_{3}^{S}=0.166\left[\mu-\mu_{6}-\mu_{5}-\mu_{4}-\mu_{2}-\mu_{1}-\mu_{3}\right] \\
& G_{4}^{S}=0.166\left[\mu-\mu_{6}-\mu_{5}-\mu_{3}-\mu_{2}-\mu_{1}-\mu_{4}\right] \\
& G_{5}^{S}=0.166\left[\mu-\mu_{6}-\mu_{4}-\mu_{3}-\mu_{2}-\mu_{1}-\mu_{5}\right] \\
& G_{6}^{S}=0.166\left[\mu-\mu_{5}-\mu_{4}-\mu_{3}-\mu_{2}-\mu_{1}-\mu_{6}\right]
\end{aligned}
$$

Para los cuatro estados de la frontera sur de Estados Unidos, la descomposición se realiza de la siguiente forma:

$$
\begin{aligned}
& G_{4}^{s}=0.25\left[\mu-\mu_{4}-\mu_{3}-\mu_{2}-\mu_{1}\right] \\
& G_{4}^{s}=0.25\left[\mu-\mu_{4}-\mu_{3}-\mu_{1}-\mu_{2}\right] \\
& G_{4}^{s}=0.25\left[\mu-\mu_{4}-\mu_{2}-\mu_{1}-\mu_{3}\right] \\
& G_{4}^{s}=0.25\left[\mu-\mu_{3}-\mu_{2}-\mu_{1}-\mu_{4}\right]
\end{aligned}
$$

Las ecuaciones previas conforman la población con medias de salario $\left[\mu_{1}, \mathrm{~K}, \mu_{6}\right]$ y $\left[\mu_{1}, \ldots ., \mu_{4}\right]$ que aíslan el efecto medio del ingreso $\boldsymbol{\mu}$ y del resto de medias en cada subgrupo, asignando el peso correspondiente. Así, la descomposición se representa con dos componentes entre e intra, $G_{k}$ que es equivalente al cálculo del índice de Gini de las ecuaciones expresadas en (1a) a (1f) y (2a) a (2d) con la siguiente ecuación:

$$
G_{k}=G_{\text {entre }}^{S}+G_{\text {intra }}^{S}
$$

En la expresión (3) la desigualdad se descompone en dos elementos, entre-grupos e intra-grupos, o al interior de los grupos (estados). 


\subsection{Resultados.}

En el cuadro 14 se muestran los resultados de la descomposición exacta del índice de Gini para la frontera norte de México, los subgrupos corresponden a los seis estados de la frontera, esto con el objetivo de analizar la participación en la desigualdad de cada uno de ellos. El primer dato que se tiene que destacar es la reducción en la desigualdad entre los estados de la frontera norte que se observa en 1994 y 2014, debe tenerse en cuenta que el análisis se realiza únicamente con ingresos provenientes del trabajo, por esta razón los índices podrían ser distintos a los Ginis reportados por instituciones oficiales.

Existen diversas razones por las que se ha presentado una reducción de la desigualdad salarial en la frontera norte de México, de acuerdo con Ochoa, Huesca y Calderón (2015) dicha reducción se atribuye a una caída del salario de los trabajadores calificados, lo que ha provocado una reducción en las disparidades salariales.

Sin embargo, de acuerdo con los resultados obtenidos en este artículo, el componente que explica en mayor medida la desigualdad salarial en la zona fronteriza del norte de México es la que se da al interior de cada estado. En 1994 la mayor participación en la desigualdad se dio en Nuevo León. En 2014 todas las participaciones se vuelven más equitativas, y la mayor participación en la desigualdad la tiene el estado de Chihuahua con un $22.40 \%$.

\section{Cuadro 14. Descomposición de la desigualdad salarial} en la frontera norte de México

\begin{tabular}{|c|c|c|c|c|c|c|}
\hline & \multicolumn{3}{|c|}{1994} & \multicolumn{3}{|c|}{2014} \\
\hline Contribución y grupo & Absoluto & Relativo & $\%$ intra & Absoluto & Relativo & $\%$ intra \\
\hline Entre & 0.038 & 0.086 & & 0.0333 & 0.1032 & \\
\hline Intra & 0.399 & 0.914 & & 0.2901 & 0.8967 & \\
\hline Gini total & 0.437 & 1 & & 0.3234 & 1 & \\
\hline \multicolumn{7}{|c|}{ Frontera Norte de México } \\
\hline Baja California & 0.043 & 0.099 & 10.87 & $0.042 v$ & 0.1299 & 14.49 \\
\hline Coahuila & 0.078 & 0.179 & 19.57 & 0.051 & 0.1577 & 17.59 \\
\hline Chihuahua & 0.036 & 0.084 & 9.14 & 0.0649 & 0.2008 & 22.4 \\
\hline Nuevo León & 0.215 & 0.492 & 53.9 & 0.0563 & 0.1741 & 19.42 \\
\hline Sonora & 0.013 & 0.029 & 3.17 & 0.03 & 0.0928 & 10.35 \\
\hline Tamaulipas & 0.013 & 0.031 & 3.34 & 0.0457 & 0.1412 & 15.75 \\
\hline Suma intra & 0.399 & 0.914 & 100 & 0.2899 & 0.8965 & 100 \\
\hline & 0.07 & & & 0.0597 & & \\
\hline$G\left(y\left(\mu / \mu_{g}\right)\right)$ & 0.432 & & & 0.3165 & & \\
\hline
\end{tabular}

Fuente: Estimación propia con datos de ENOE 
El cuadro 15 muestra la descomposición para la frontera sur de Estados Unidos, a diferencia de lo que sucedió en México, destaca un incremento en la desigualdad total, dicho incremento se encuentra en línea con la Hipótesis del Cambio Tecnológico Sesgado (HCTS), misma que asegura que se ha incrementado la desigualdad salarial como consecuencia del impacto del cambio tecnológico en el mercado de trabajo, lo que provoca un sesgo que favorece a los trabajadores usuarios de tecnología en detrimento de los no usuarios, (Calderón, Huesca y Ochoa (2017). Durante todo el periodo de análisis el estado de California es el que contribuye en mayor medida a la desigualdad total.

\begin{tabular}{|c|c|c|c|c|c|c|}
\hline \multirow[b]{2}{*}{ Contribución a grupo } & \multicolumn{3}{|c|}{1994} & \multicolumn{3}{|c|}{2014} \\
\hline & Absoluto & Relativo & $\%$ intra & Absoluto & Relativo & $\%$ intra \\
\hline Entre & 0.004 & 0.01 & & 0.0217 & 0.0433 & \\
\hline Intra & 0.385 & 0.99 & & 0.4788 & 0.9566 & \\
\hline Gini total & 0.389 & 1 & & 0.5005 & 1 & \\
\hline \multicolumn{4}{|c|}{ Frontera Sur de Estados Unidos } & & & $G\left(\mu_{g}\right.$ \\
\hline Arizona & 0.022 & 0.057 & 5.74 & 0.0346 & 0.0693 & 7.25 \\
\hline California & 0.231 & 0.595 & 60.16 & 0.264 & 0.5274 & 55.14 \\
\hline Nuevo México & 0.006 & 0.015 & 1.54 & 0.006 & 0.012 & 1.25 \\
\hline Texas & 0.125 & 0.322 & 32.57 & 0.174 & 0.3478 & 36.36 \\
\hline Suma intra & 0.385 & 0.989 & 100 & 0.4786 & 0.9565 & 100 \\
\hline$G\left(\mu_{g}\right)$ & 0.008 & & & 0.041 & & \\
\hline$G\left(y\left(\mu / \mu_{g}\right)\right)$ & 0.389 & & & 0.498 & & \\
\hline
\end{tabular}

Fuente: Estimación propia con datos de la Encuesta Nacional de Ocupación y Empleo, INEGI.

\section{Conclusión}

A través de este análisis, se ha podido constatar que se da una convergencia de los PIB per cápita estatales de los estados colindantes de la zona fronteriza de los Estados Unidos y de México. Pero, esta convergencia se explica por el hecho de que el nivel de vida de los estados del sur de los Estados Unidos ha estado por debajo del promedio nacional de ese país; mientras que el nivel de vida de los estados del norte de México está por encima del promedio nacional. De manera que la tasa de crecimiento de los estados del sur de los Estados Unidos ha sido mucho más baja que la de los estados del norte de México. 
En lo que se refiere al nivel de desarrollo económico de los estados colindantes de la zona fronteriza de los Estados Unidos y México, se observa que, a pesar de la tendencia hacia la convergencia del PIB per cápita estatal de la zona fronteriza de ambos países, existe una brecha muy importante en los niveles de vida de la población de los estados fronterizos de uno y otros países.

Por lo que tal convergencia es relativa, ya que los niveles de desarrollo económico de ambos países mantienen una brecha muy importante en los niveles de vida de la población. La esperanza de vida en promedio es más alta en los estados norteamericanos que en los estados mexicanos y el nivel de educación promedio es mayor en los EEUU que en México. El grado de rezago social, la desigualdad del ingreso y pobreza es mayor en los Estados de la frontera norte.

Con respecto al comportamiento salarial, se observó que mientras los salarios promedio de los estados del sur de los estados unidos tendieron a aumentar de manera significativa entre 1994 y el 2014. Sobre todo, en los estados de Arizona y Nuevo México. En México cayeron abruptamente más de 100\%

La caída tan drástica de los salarios en los estados de la Frontera Norte de México se observa una reducción en las desigualdades salariales, que Ochoa, Huesca y Calderón (2015) atribuyen a la caída del salario de los trabajadores calificados, lo que ha provocado una reducción en las disparidades salariales. De acuerdo con nuestros resultados, encontramos que es sobre todo la desigualdad al interior de los estados la que explica esta reducción de la desigualdad. En el caso de la frontera norte de México se observa que en 1994 la mayor participación en la desigualdad es del estado de Nuevo León, y que en 2014 todas las participaciones se vuelven más equitativas, y la mayor participación en la desigualdad la tiene el estado de Chihuahua.

En el caso de los estados de la frontera sur de los EE.UU. los que más contribuyeron a la desigualdad salarial fueron los estados de california y Texas donde se pagan los salarios más altos

\section{Referencias}

Acemoglu, Daron y David Autor. 2011. Skills, tasks and technologies: Implications for employment and earnings. En Handbook of Labor Economics, editado por Orley Ashenfelter y David Card. Amsterdam: North Holland, 1043-1171.

Anderson, Joan y James Gerber. 2008. Fifty years of change on the U.S. -Mexico border. Texas: University of Texas Press.

Bhattacharya, Nath y B. Mahalanobis. 1967. Regional disparities in household consumption in India. Journal of the American Statistical Association, 62 (317): 143-161.

Calderón, Cuauhtémoc, Luis Huesca y Gloria Ochoa. 2017. Análisis comparativo de la desigualdad salarial entre México y Estados Unidos. Investigación Económica, 76 (300): 3-31.

Consejo Nacional de Evaluación de la Política de Desarrollo Social. 2017. http://www.coneval.org.mx/Paginas/principal.aspx (09 de octubre, 2017).

Consejo Nacional de Población. 2017. https://www.gob.mx/conapo (11 de octubre, 2017) .

Current Population Survey (CPS). 2017. U.S. Census bureau, current population survey, annual social and economic supplement. https://www.census.gov/programs-surveys/cps.html (12 de septiembre, 2017).

Encuesta Nacional de Empleo Urbano. 2017. http://www.beta.inegi.org.mx/proyectos/enchogares/historicas/ene/default.html (12 de septiembre, 2017). 
Encuesta Nacional de Ocupación y Empleo. 2017. http://www3.inegi.org.mx/sistemas/temas/default. aspx? $\mathrm{s}=\mathrm{est} \& \mathrm{c}=25433 \& \mathrm{t}=1$ (12 de septiembre, 2017).

Huesca, Luis y Gloria Ochoa. 2016. Desigualdad salarial y cambio tecnológico en la frontera norte de México. Problemas del Desarrollo, 47 (187): 165-188.

Kolenikov, Stanislav y Anthony Shorrocks. 2005. A decomposition analysis of regional poverty in Russia. Review of Development Economics, 9 (1): 25-46.

Morduch, Jonathan y Terry Sicular. 2002. Rethinking inequality decomposition, with evidence from rural China. Economic Journal, 112 (476): 93-106.

Ochoa, Gloria, Luis Huesca y Cuauhtémoc Calderón. 2015. Descomposición de la desigualdad salarial en los estados de la frontera norte de México. Economía Informa, (393): 3-20.

Population Division, Banco Mundial. 2017. https://datos.bancomundial.org/indicador/SP.POP. GROW?locations=LB\&display=graph (12 de septiembre, 2017).

Prebisch, Raúl. 1971. Transformación y desarrollo: La gran tarea de América Latina. Chile: Cepal, Naciones Unidas.

Rodríguez, Reyna, Luis Huesca y Mario Camberos. 2011. Mercado laboral, inequidad salarial y cambio tecnológico. Frontera Norte, 23 (45): 7-33.

Shapley, Lloyd. 1953. A value for N-person games. En Contributions to the theory of game, editado por Harold Khun y Albert Tucker. Princeton: Princeton University Press, 307-317.

Shorrocks, Anthony. 1982. Inequality decomposition by factor components. Econometrica: Journal of the Econometric Society, 50(1): 193-211.

Shorrocks, Anthony. 2013. Decomposition procedures for distributional analysis: a unified framework based on the shapley value. Journal of Economic Inequality, 11(1): 99-126.

Sistema de Cuentas Nacionales de México. 2017. http://www.inegi.org.mx/default.aspx (14 de octubre, 2017). Tamayo, Jesús y José Luis Fernández. 1983. Zonas fronterizas (México-Estados Unidos). Ciudad de México: CIDE

Torres, Aldo y Gustavo Félix. 2017. Rendimiento al uso de PC en México: un análisis comparativo por sector de actividad. https://www.researchgate.net/publication/322644664_El_uso_de_Internet_y_su_relacion_con_los_salarios_en_Mexico_un_analisis_no_parametrico (10 de marzo. 2017).

US Departament of Commerce, Bureau of Economic Analysis. 2017. https://www.bea.gov/ (12 de septiembre, 2017).

US Department of Commerce, Bureau of Economic Analysis, U.S. Census Bureau. 2017. https://blog.bea. gov/category/u-s-census-bureau/ (12 de septiembre, 2017). 